\title{
Impact of food processing on rye product properties and their in vitro digestion
}

\author{
Daniel P. Johansson ${ }^{1} \cdot J^{\prime}$ sé L. Vázquez Gutiérrez ${ }^{1}$ - Rikard Landberg ${ }^{1,2,3}$ • \\ Marie Alminger $^{3} \cdot$ Maud Langton $^{1}$
}

Received: 30 September 2016 / Accepted: 7 April 2017 / Published online: 17 April 2017

(C) The Author(s) 2017. This article is an open access publication

\begin{abstract}
Purpose Rye products have been reported to elicit postprandial insulin and glucose responses which may be beneficial for prevention of type- 2 diabetes. However, mechanisms underlying variations in responses related to processing techniques are not fully understood.

Methods Five differently processed rye products (sourdough-fermented bread, fermented and unfermented crispbread, extrusion-cooked rye, and porridge) and refined wheat bread were characterised. Two in vitro methods, a dynamic method simulating digestion in the stomach and small intestine and a static method, simulating conditions in the stomach were used to determine viscosity development, structural changes and release of glucose during digestion.

Results Structural and compositional differences induced by processing influenced product digestion. Gastric disintegration and digesta particle size were related to characteristics of the starch/protein matrix, while digesta viscosity was reduced due to fibre degradation during fermentation. More cohesive boluses were associated with slower glucose release. Sourdough fermentation increased amylose leakage and appeared to inhibit starch hydrolysis despite low digesta viscosity and rapid disintegration.
\end{abstract}

Daniel P. Johansson

daniel.p.johansson@slu.se

1 Department of Molecular Sciences, Swedish University of Agricultural Sciences (SLU), Uppsala, Sweden

2 Nutritional Epidemiology Unit, Institute of Environmental Medicine, Karolinska Insitutet, Stockholm, Sweden

3 Department of Biology and Biological Engineering, Food and Nutrition Science, Chalmers University of Technology, Gothenburg, Sweden
Conclusions The net release of glucose during digestion of foods is determined by several factors which may vary in their importance depending on product specific properties.

Keywords In vitro digestion - Glucose release ·

Microstructure · Dietary fibre - Gastric digestion · Rye

\section{Introduction}

High postprandial glucose and insulin concentrations in the blood may induce oxidative stress and low-grade inflammation [1], and result in decreased insulin sensitivity and subsequent increased risk of developing type-2 diabetes [2]. Foods which elicit beneficial glucose and hormonal responses may, therefore, help prevent cardiovascular diseases and type- 2 diabetes.

Rye (Secale cereal L.) is an important crop in Eastern, Central and Northern Europe and a rich source of dietary fibre [3]. In acute intervention studies on healthy subjects, rye products have repeatedly been shown to induce lower postprandial insulin response, with or without a corresponding decrease in glucose response, compared with refined wheat bread [4-7]. The underlying mechanisms are not known, but have been suggested to relate to structural features of rye products, such as dense structure and formation of an amylose layer around starch granules, inhibiting starch hydrolysis [4]. In addition, high content and molecular weight of certain fibres in rye could influence glucose absorption rate and hormonal responses through decreased diffusion rates of nutrients $[8,9]$. In a recent study, unfermented whole grain rye crispbread was observed to induce a lower insulin response than a corresponding fermented product [5]. This was attributed partly to degradation of 
$\beta$-glucan and arabinoxylan by endogenous enzymes during fermentation.

Other processes and processing conditions may also affect starch hydrolysis and postprandial glucose and insulin responses to rye products, but comparative studies are lacking. In cereal-based foods, botanical integrity, e.g., cracked grains compared with flour, decreases the release rate of nutrients by limiting access by enzymes [10]. Extrusion cooking with high temperatures and mechanical forces results in loss of structural integrity and a high degree of starch gelatinisation, which can increase in vitro digestibility of starches [11]. Water availability, cooking time and temperature also affect starch gelatinisation, with implications for the rate and extent of hydrolysis [12]. Moreover, dense food structure may decrease the rate of starch hydrolysis and the postprandial glucose and insulin responses, as shown for pasta compared with white bread [13]. Furthermore, while fibre degradation during fermentation may increase the rate of starch hydrolysis and absorption, organic acids produced during sourdough fermentation may counteract this through inhibition of starch hydrolysis and reduced gastric emptying [14].

Although human studies are usually considered the most reliable approach for accurate prediction of release and absorption of nutrients through the gut wall, establishment of mechanistic relationships is complicated. For example, the concentration of glucose in circulation is not a direct reflection of absorption, as it also depends on endogenous production rate and clearance rate [15], which are regulated by both insulin and the incretins GLP-1 and GIP, released in response to nutrient absorption [16]. In vitro methods (static or dynamic) are appropriate for mechanistic studies and widely used to predict the behaviour of food components in the digestive tract. Release of maltose and microstructural changes in food products during digestion has been studied previously using the dynamic in vitro model TNO Gastro-Intestinal Model (TIM) [17].

Most studies to date have focused on one product type, e.g., bread or extruded cereals, and how specific processing parameters or different raw materials affect in vitro digestion and postprandial responses in humans. Although different product types based on the same raw material have been compared, understanding of how differences in their properties and how they are digested and may contribute to postprandial responses, is limited. The aim of this study was to investigate how processing influences the microstructure and composition of rye products, the digesta properties and release of glucose during in vitro digestion. Five differently processed rye products, based on the same raw material, but differing in microstructure regarding the protein/starch matrix and the distribution and integrity of dietary fibre were therefore selected and compared with refined wheat bread. The results may also be of relevance when considering release of other compounds in cerealbased foods.

\section{Materials and methods}

\section{Products}

Of the six products included in the study, three were commercially available: refined wheat bread (WB) (Pågen $A B$, Sweden), yeast-fermented whole grain rye crispbread (RCB) and unfermented whole grain rye crispbread (uRCB) (Barilla Sweden AB, Sweden). Flour of the same origin and composition, but milled to different particle sizes, was used for production of RCB and uRCB. According to data provided by the manufacturer, the uRCB flour had 30-42\% particles $<125 \mu \mathrm{m}$ and $20-28 \%>1040 \mu \mathrm{m}$, while the corresponding proportions in the RCB flour were $51-57 \%$ and 3-6\%, respectively. RCB was fermented in two steps; first $120 \mathrm{~min}$ at $29^{\circ} \mathrm{C}$, followed by $35 \mathrm{~min}$ with an increase from 30 to $38{ }^{\circ} \mathrm{C}$. For uRCB, flour was mixed with water at $12{ }^{\circ} \mathrm{C}$ and then whipped at $6{ }^{\circ} \mathrm{C}$ to incorporate air into the batter.

The other three products were produced in-house, using the same flour as for RCB. Sourdough-fermented whole grain rye bread (sRB) was prepared by mixing $5150 \mathrm{~g}$ whole grain rye flour, $35.9 \mathrm{~g} \mathrm{NaCl}, 1300 \mathrm{~g}$ commercial sourdough (Jästbolaget $\mathrm{AB}$, Sweden), $125 \mathrm{~g}$ fresh yeast and $4405 \mathrm{~g} \mathrm{H}_{2} \mathrm{O}$. The dough was mixed for $4 \mathrm{~min}$ at low speed and $4 \mathrm{~min}$ at high speed in a dough mixer (Varimixer, Charlotte, NC, USA), proofed for $30 \mathrm{~min}$ at room temperature, divided into $900 \mathrm{~g}$ portions and placed in baking tins, and then put in a proofing chamber at $38{ }^{\circ} \mathrm{C}$ and $80 \%$ relative humidity for $40 \mathrm{~min}$. Baking (50 min) was initiated at $230{ }^{\circ} \mathrm{C}$ with $8 \mathrm{~s}$ of steam, and then immediately lowered to $190^{\circ} \mathrm{C}$.

Extruded whole grain rye (extR) was produced at VTT Technical Research Centre of Finland (Espoo, Finland). Whole grain flour, with addition of $0.8 \% \mathrm{NaCl}$, was extruded using a co-rotating twin-screw extruder APV MPF 19/25 with conveying and mixing elements (Baker Perkins Group Ltd, Peterborough, UK). The flour feed rate was $50 \mathrm{~g} / \mathrm{min}$, water addition $3.0 \mathrm{ml} / \mathrm{min}$, screw speed $400 \mathrm{rpm}$ and in-barrel residence time around $2 \mathrm{~min}$. Die pressure was measured using a pressure transducer (Dynisco Ltd., UK) in the die plate. The temperature profile of the four heating blocks was: $140-100-80-60{ }^{\circ} \mathrm{C}$ (from die exit to feeding section). The parameters measured were: torque $76-86 \%$ (of maximum $100 \%$ ), pressure at die 3.9-4.4 bar and temperature at die $124{ }^{\circ} \mathrm{C}$. The expanded products were cut by the cutter blade (in front of the die exit) into breakfast cereal spheres (5-7 $\mathrm{mm}$ in diameter) and ovendried at $80^{\circ} \mathrm{C}$ for $15 \mathrm{~min}$. 
Whole grain rye porridge (RP) was prepared by mixing $64 \mathrm{~g}$ whole grain rye flour and $0.58 \mathrm{~g} \mathrm{NaCl}$ with $200 \mathrm{~g}$ boiling water and stirring with a spoon for $2 \mathrm{~min}$ to ensure good mixing. The porridge was allowed to rest for $3 \mathrm{~min}$ before use.

Salt content in the rye products was equal and based on the content in the commercially produced rye crispbreads. WB had a higher content of salt $(1.5 \% \mathrm{db}$.).

\section{Chemical analysis of products}

Samples were milled with a cyclone sample mill (Retsch, Haan, Germany). Samples with high water content (WB, $\mathrm{sRB}, \mathrm{RP}$ ) were freeze-dried prior to milling. RP was prepared as described above and immediately frozen in liquid nitrogen before freeze-drying. Extractable and unextractable dietary fibre content and composition were analysed according to the Uppsala method [18]. Content of arabinoxylan and arabinogalactan was calculated assuming an arabinose/galactose ratio of 0.69 in extractable arabinogalactan [19]. $\beta$-glucan, fructan and resistant starch content was analysed using a K-BGLU kit [20], a K-FRUC kit [21], and a K-RSTAR kit [22], respectively (Megazyme, Bray, Ireland). Calcofluor average molecular weight of $\beta$-glucan $\left(\mathrm{M}_{\mathrm{cf}}\right)$ was analysed using size exclusion chromatography with fluorescence detection [23]. Crude fat was determined according to the method described in the Official Journal of the European Communities [24] and protein according to the Kjeldahl method $(\mathrm{N} \times 6.25)$ [25]. Dry content was determined by drying the samples at $105{ }^{\circ} \mathrm{C}$ for $16 \mathrm{~h}$ according to AACC method 44-15A.

\section{In vitro digestion}

\section{Mastication}

Mastication of samples for the in vitro trials was conducted by one subject according to the method used by Ballance et al. [26]. For use in the TIM model, all expectorated samples were collected. For the simulated gastric digestion, due to the small sample volume used, the first three expectorated boluses were discarded to allow stabilisation of salivary flow and mastication behaviour [27]. The sample mass collected for the in vitro trials was based on expected dry matter content of sample boluses, as determined in three replicates per product. Dry matter content of the boluses used for each experiment was also determined.

\section{Static gastric digestion and viscosity}

An adaptation of the standard method proposed within the COST action Infogest was used for static gastric in vitro digestion [28]. Boluses were collected as described above and water added to give the same dry content in all samples (approximately 25\%). To the bolus/water mixture, simulated gastric fluid, prepared as described by Minekus et al. [28], was added to give a final ratio of 1:1 (bolus/ water mixture:simulated gastric fluid, dry matter content approximately $12.5 \%$ ) after adjustment of $\mathrm{pH}$ to 3 . Using a total volume of $25 \mathrm{ml}$, gastric digestion was then performed in a Rapid Visco Analyser (RVA) (Newport Scientific Pvt. Ltd., Australia) at a rate of $120 \mathrm{rpm}$, temperature $37^{\circ} \mathrm{C}$ and 2-h run time, to monitor the development of viscosity during gastric digestion. Viscosity data was collected over 1-s periods. A standard RVA paddle was used, but the cup was modified by addition of three vertically attached plastic baffles $(1 \mathrm{~mm} \times 1 \mathrm{~mm} \times 50 \mathrm{~mm})$ evenly distributed on the inside of the cup. The addition of baffles was required to facilitate mixing and prevent the bolus from being dragged by the paddle. This gave a minimum gap of approximately $1 \mathrm{~mm}$ between the paddle and the baffles (standard gap is $1.9 \mathrm{~mm}$ between paddle and cup wall). The final viscosity, i.e., after $120 \mathrm{~min}$, was used for viscosity comparison. Sodium bicarbonate was added to the remaining sample to neutralise the $\mathrm{pH}$ and stop pepsin activity, and the samples were stored at $4{ }^{\circ} \mathrm{C}$ before particle size determination on the same day. All samples were run in triplicate. Order of samples and replicates was randomised.

\section{Particle size distribution}

Samples collected from static in vitro gastric digestion were passed through a series of sieves with decreasing mesh size (3150, 2000, 1000, 600, 425 and $250 \mu \mathrm{m})$. Each sieve was carefully rinsed for $30 \mathrm{~s}$ with cold water before removal from the sieve stack to reduce the number particles of sizes below the mesh size retained. Excess water was then removed from the fraction retained in each sieving step by filtration and dry matter content of the remaining sample was determined. Particle size fractions are reported as a percentage of total dry weight.

\section{$T I M$}

The dynamic in vitro model TIM (TNO, Zeist, Netherlands) was used for simulating digestion in the stomach and small intestine (Fig. 1). This model consists of a series of compartments representing the stomach, duodenum, jejunum and ileum [29]. Solutions were prepared according to Salovaara et al. [30]. A medium meal transit time was chosen to simulate a semi-solid meal, and the half-time of stomach emptying was $70 \mathrm{~min}$. The $\mathrm{pH}$ was measured every min and $\mathrm{HCl}$ was secreted into the gastric compartment to gradually decrease $\mathrm{pH}$ over time $(\mathrm{pH} /$ time $(\mathrm{min})$ : $6 / 0 ; 5.7 / 15 ; 4.5 / 45 ; 2.9 / 90 ; 2.3 / 120 ; 1.8 / 240 ; 1.6 / 300)$. $\mathrm{NaHCO}_{3}$ was secreted in the intestinal compartments to 


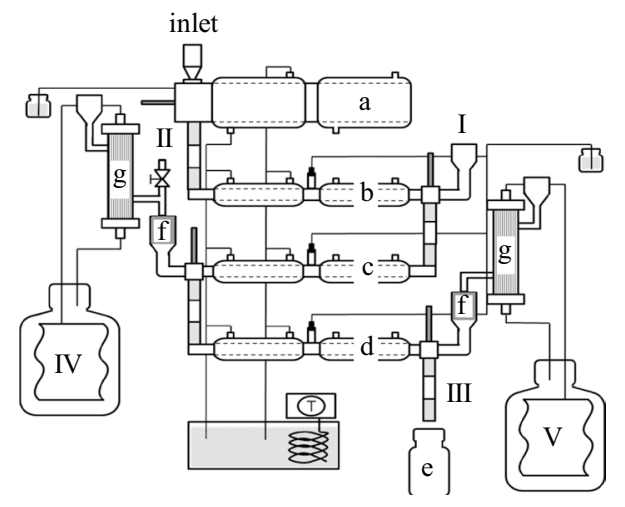

Fig. 1 Schematic diagram of the TNO gastroi-intestinal model (TIM): a stomach, $\mathbf{b}$ duodenum, $\mathbf{c}$ jejunum, $\mathbf{d}$ ileum, $\mathbf{e}$ ileal delivery, $\mathbf{f}$ pre-filters, $\mathbf{g}$ dialysis filters. Roman numerals indicate sample collection sites: $I$ ) duodenum, $I I$ ) jejunum, $I I I$ ) ileocaecal valve, $I V$ ) dialysis jejunum, $V$ ) dialysis ileum

hold the $\mathrm{pH}$ on the set-point levels at approximately 6.5 in duodenum and 6.5-7-7.5 in jejunum and ileum. The same protocol was used for all products.

Mastication was performed as described above and boluses equal to a mass containing $20 \mathrm{~g}$ starch were collected. Water was added to give a total mass of $300 \mathrm{~g}$ (dry content $9-10 \%)$ and the product was fed into the stomach compartment of the model. The total time from end of mastication to initiation of in vitro digestion was $5 \mathrm{~min}$. The in vitro digestion was performed in triplicate for $\mathrm{WB}, \mathrm{sRB}$ and RP and in duplicate for RCB, uRCB and extR. Samples and replicates were run in randomised order.

Samples for glucose and maltose analysis were collected from the jejunum (II in Fig. 1) at 15, 30, 45, 60, 90, 120 and $180 \mathrm{~min}$ and boiled for $10 \mathrm{~min}$ to stop amylase activity. The samples were then immediately frozen at $-20{ }^{\circ} \mathrm{C}$ and at the end of each day moved to a freezer at $-80{ }^{\circ} \mathrm{C}$ for storage until analysis.

\section{Glucose and maltose analysis}

Prior to analysis, samples collected from the TIM model were thawed, centrifuged and diluted 200- to 2000-fold. Glucose and maltose concentrations were analysed using a Dionex HPAEC system (Dionex, Sunnyvale, CA, USA) consisting of a CarboPak PA-1 ion exchange column $(4 \times 250 \mathrm{~mm}$ with guard $4 \times 50 \mathrm{~mm})$ coupled with a pulsed amperometric detector. The mobile phase was run isocratically with a mixture of $95 \% 150 \mathrm{mM} \mathrm{NaOH}(\mathrm{A})$ and $5 \% 150 \mathrm{mM} \mathrm{NaOH}$ with $500 \mathrm{mM} \mathrm{NaOAc}$ (B) for $8 \mathrm{~min}$ for elution of glucose and maltose, and then increased to $100 \%$ $\mathrm{B}$ and maintained at $100 \% \mathrm{~B}$ for 6 min for elution of soluble carbohydrates of higher molecular weight. The mobile phase mixture was then reset to $95 \% \mathrm{~A}$. The flow rate was $1 \mathrm{ml} / \mathrm{min}$ and total run time for each sample $20 \mathrm{~min}$.
Quality control samples run with each batch gave withinbatch and between-batch variation of 1.4-3.6 and 4.4\%, respectively. The sum of glucose and maltose recalculated to glucose was used for statistical analysis and is referred to as glucose in the text.

\section{Microscopy}

Samples for microstructural characterisation were collected from the products, after mastication, after static simulated gastric digestion and in the TIM model from the duodenum (I in Fig. 1) at 30, 60, 90, 120 and 180 min, and from the ileal delivery (III in Fig. 1) at 60, 90, 120, 180, 240 and $360 \mathrm{~min}$. All samples, except product samples to be embedded in plastic, were frozen in liquid nitrogen on collection and stored at $-80{ }^{\circ} \mathrm{C}$ until analysis. Image $\mathrm{J}$ (fiji.sc/Fiji) was used for processing of micrographs and figures.

Immunolocalisation by confocal laser scanning microscopy (CLSM)

Frozen product samples were embedded in optimal cutting temperature compound and cut into $10 \mu \mathrm{m}$ thick sections in a Leica CM1860 cryostat (Leica, Austria). The sections were incubated for $40 \mathrm{~min}$ in PBS buffer solution $\left(0.1 \mathrm{M} \mathrm{Na}_{2} \mathrm{HPO}_{4}, 0.1 \mathrm{M} \mathrm{NaH}_{2} \mathrm{PO}_{4}, 0.15 \mathrm{M} \mathrm{NaCl}, \mathrm{pH} 7.2\right)$ containing $1.5 \%$ bovine serum albumin (BSA) to prevent non-specific binding. The sections were then incubated for $2 \mathrm{~h}$ at $25^{\circ} \mathrm{C}$ with primary antibody solution. Monoclonal antibodies raised against $(1 \rightarrow 3,1 \rightarrow 4)-\beta$-D-glucan (Biosupplies, Parkville, Australia) and arabinoxylan (LM11 antibody, Plant Probes, Leeds, UK) were diluted 1:100 and 1:50, respectively, in a PBS buffer solution containing $0.4 \%$ BSA. Appropriate controls were made by replacing the primary antibody solution with PBS buffer containing 0.4\% BSA. After incubation, the sections were rinsed with PBS solution and incubated again for 40 min with PBS buffer solution containing $0.4 \%$ BSA. They were then incubated for $2 \mathrm{~h}$ in darkness with fluorescently labelled secondary antibodies, i.e. Alexa Fluor ${ }^{\circledR} 488$ goat anti-mouse IgG $(\mathrm{H}+\mathrm{L})$ and Alexa Fluor ${ }^{\circledR} 568$ goat anti-rat $\mathrm{IgG}(\mathrm{H}+\mathrm{L})$ for BG and arabinoxylan, respectively, and finally rinsed with PBS and water. To achieve double immunolabelling, the whole procedure was carried out with anti-BG and Alexa Fluor $^{\circledR} 488$ antibodies and then repeated on the same section with LM11 and Alexa Fluor ${ }^{\circledR} 568$ antibodies.

Micrographs were acquired using a Zeiss LSM 780 confocal microscope (Carl Zeiss, Germany) with a PlanApochromat $20 \times / 0.8$ M27 objective. A $488 \mathrm{~nm}$ Argon laser and a $561 \mathrm{~nm}$ diode pumped solid state laser were used as excitation sources and fluorescence emissions were collected between 493-578 $\mathrm{nm}$ and 570-640 nm. 
Zen2012 software (Carl Zeiss, Germany) was used in acquisition.

\section{Bright field microscopy}

Samples were fixed in 5\% glutaraldehyde in $0.1 \mathrm{M}$ phosphate buffer ( $\mathrm{pH} \mathrm{6.8)} \mathrm{for} 12 \mathrm{~h}$, washed with phosphate buffer, fixed for another $2 \mathrm{~h}$ in $4 \% \mathrm{OsO}_{4}$, and again washed in phosphate buffer. Samples were then dehydrated in a series of aqueous ethanol of increasing concentration, and finally infiltrated and polymerised with Technovit 7100. In vitro digested samples were embedded in cold gelling agar prior to the dehydration steps. To ensure representative samples of the masticated and digested samples, embeddings were made for each replicate and, in TIM, at each time point. Time points used for TIM were, after an initial screening, limited to: 60, 90 and 120 min from duodenum and 90, 120 and 180 from ileal delivery. A minimum volume of $0.5 \mathrm{ml}$ of digesta was used for each embedding.

The embedded samples were cut into $1.5 \mu \mathrm{m}$ thick sections with an ultra-microtome (Leica EM UC6, Leica, Austria) and stained with Lugol's solution. The stained sections were examined using a Nikon Eclipse Ni-U microscope with $4 \times / 0.20$ and $40 \times / 0.95$ Plan-Apochromat objectives and images captured with a Nikon Digital Sight DS-Fi2 camera and processed with the software NIS-Elements BR (Nikon Instruments Europe, Amsterdam, Netherlands).

\section{Statistical analysis}

Statistical analysis was conducted using SAS version 9.4 (SAS Institute Inc., Cary, NC, USA). Differences between glucose profiles were evaluated with a mixed effect model, PROC mixed, suitable for repeated measurements. For glucose profiles, separate models were used for concentration-time profiles and area under the curve (AUC). AUC was calculated using the trapezoid rule for the intervals between $0-90$ and $0-180 \mathrm{~min}$. For concentration models, time, product and a time $\times$ product interaction term were used as fixed effects, with time as a repeated variable. Run order was included as a random factor. When significant time $\times$ product interactions were found, pair-wise comparisons were performed at these time points. For AUC models, only product and run order were included in the model and pair-wise comparisons made. Differences in final viscosity between samples were evaluated using one-way ANOVA. All pairwise comparisons were made using Tukey's honest significance test.

\section{Results}

\section{Chemical characterisation}

Water content (\% wb) was 35.7 and $53.0 \%$ for the soft breads WB and sRB, 7.5, 8.6 and $8.0 \%$ for the dry products RCB, uRCB and extR, and $77.0 \%$ for the semi-solid $\mathrm{RP}$. The protein, fat and starch content were higher in WB than in the rye products, while the rye products contained more fibre (Table 1). Total fibre content was comparable between the rye products, but fibre composition varied. $\beta$-glucan and arabinoxylan differed only slightly in content, while average molecular weight $\left(\mathrm{M}_{\mathrm{cf}}\right)$ of $\beta$-glucan was lower in the fermented $\mathrm{SRB}$ and RCB than in the unfermented $\mathrm{uRCB}$, extR and RP. $\beta$-glucan extractability was higher in the extruded rye product than in the other unfermented products. The extractable arabinoxylan content followed a similar pattern, being lowest in uRCB and RP. Resistant starch was highest in SRB and lowest in extR, but relatively similar in the other products.

\section{Product microstructure}

Differences between the products in terms of structure and distribution of $\beta$-glucans and arabinoxylans were observed in the CLSM micrographs (Fig. 2). $\beta$-Glucan was distributed as smaller fragments throughout the matrix in the fermented $\mathrm{WB}$, sRB and RCB, compared with the unfermented uRCB, extR and RP. Arabinoxylan followed a similar pattern, but was also distributed throughout the starch matrix in RP. In extR, $\beta$-glucan and arabinoxylan were clearly separated and distributed as smaller fragments throughout the matrix. The cell walls in RP appeared more swollen than in the other products.

In WB, protein formed a continuous network, encapsulating starch granules, while all rye products had a continuous starch network encapsulating protein (Fig. 3). For RCB and uRCB, the starch phase consisted of highly swollen starch granules. In uRCB the granules were often indistinguishable, indicating a higher degree of starch gelatinisation than in RCB. The lamella also appeared thinner in uRCB than in RCB. Furthermore, uRCB contained larger pieces of bran and intact cell structures compared with the other rye products. In sRB, starch granules were less swollen and surrounded by a layer of leaked amylose. Leaked amylose was also observed in uRCB and, to a lesser extent, in RCB. In extR all starch granules were completely disrupted, resulting in a homogeneous starch phase, which encapsulated small fragments of cell walls and aleurone layers. The thickness of the lamella in extR was comparable to that in uRCB. In RP, 
Table 1 Product composition (\%, dry basis) and calcofluor average molecular weight $\left(\mathrm{M}_{\mathrm{cf}}\right)$ of extractable $\beta$-glucan

\begin{tabular}{|c|c|c|c|c|c|c|}
\hline & WB & sRB & $\mathrm{RCB}$ & $\mathrm{uRCB}$ & ExtR & $\mathrm{RP}$ \\
\hline Protein & 11.8 & 8.7 & 9.6 & 9.6 & 8.3 & 8.3 \\
\hline Fat & 6.7 & 1.6 & 1.7 & 1.6 & 1.6 & 1.7 \\
\hline Available carbohydrates ${ }^{\mathrm{a}}$ & 73.4 & 67.2 & 68.0 & 66.0 & 68.9 & 67.1 \\
\hline Resistant starch & 1.8 & 3.3 & 0.8 & 0.7 & 0.1 & 1.2 \\
\hline Ash & 2.2 & 2.3 & 2.4 & 2.3 & 2.3 & 2.2 \\
\hline \multicolumn{7}{|l|}{ Dietary fibre } \\
\hline Total $^{\mathrm{b}}$ & 6.0 & 20.2 & 18.3 & 20.5 & 18.9 & 20.7 \\
\hline Extractable $^{c}$ & 1.8 & 6.8 & 7.2 & 7.9 & 8.7 & 7.6 \\
\hline Unextractable & 4.2 & 13.4 & 11.1 & 12.6 & 10.2 & 13.1 \\
\hline \multicolumn{7}{|l|}{ Arabinoxylan $^{\mathrm{d}}$} \\
\hline Total & 1.9 & 8.5 & 8.6 & 8.8 & 8.4 & 8.7 \\
\hline Extractable & 0.9 & 3.0 & 3.0 & 2.6 & 3.4 & 2.5 \\
\hline Unextractable & 1.0 & 5.6 & 5.6 & 6.3 & 5.1 & 6.2 \\
\hline Arabinogalactan & 0.2 & 0.2 & 0.2 & 0.1 & 0.1 & 0.2 \\
\hline$\beta$-glucan & 0.3 & 2.1 & 2.1 & 2.5 & 2.1 & 2.1 \\
\hline Cellulose and resistant starch ${ }^{\mathrm{e}}$ & 2.4 & 4.6 & 2.5 & 2.7 & 2.1 & 3.2 \\
\hline Fructan & 0.4 & 2.5 & 2.6 & 4.0 & 3.9 & 4.2 \\
\hline Klason lignin & 0.1 & 1.5 & 1.3 & 1.3 & 1.3 & 1.5 \\
\hline$\beta$-glucan $\mathrm{M}_{\mathrm{cf}}\left(10^{5} \mathrm{~g} / \mathrm{mol}\right)$ & 1.5 & 2.5 & 1.4 & 4.5 & 6.5 & 7.3 \\
\hline Extractability, $\beta$-glucan (\%) & 17 & 27 & 27 & 18 & 36 & 16 \\
\hline
\end{tabular}

$W B$ refined wheat bread. $S R B$ sourdough-fermented whole grain rye bread. $R C B$ yeast-fermented whole grain rye crispbread. $u R C B$ unfermented whole grain rye crispbread. extR extruded whole grain rye. $R P$ whole grain rye porridge

${ }^{a}$ Calculated by difference (total minus fat, protein, fibre and ash)

b Calculated as the sum of fructan and total dietary fibre, as analysed by the Uppsala method [18]

c Calculated as the sum of fructan and total extractable dietary fibre, as analysed by the Uppsala method [18]

${ }^{\mathrm{d}}$ Calculated from the sum of arabinose, xylose and galactose, assuming an arabinose to extractable galactose ratio of 0.69 in arabinogalactan [19]

e Calculated as the difference between total $\beta$-glucan and glucose residues, as analysed by the Uppsala method [18]

larger fragments, consisting of aleurone layers and starch granules encapsulated in intact cells, were separated by a continuous phase consisting of loose starch granules in a dilute phase of leaked starch.

\section{Mastication and simulated gastric digestion}

The number of chewing cycles before expectoration varied between the products: 3-5 for RP, 10-15 for extR, 15-20 for WB, 20-25 for sRB and 25-30 for RCB and uRCB. Bolus water content $(\% \mathrm{wb}$, standard deviation in brackets) was $51.6 \%$ (2.0), 49.2\% (2.2), $48.9 \%$ (1.9) and $47.2 \%$ (1.5) for WB, RCB, uRCB and extR, respectively, $60.5 \%$ (1.3) for sRB and $75.1 \%(0.1)$ for RP. During mastication, the protein/starch matrix of WB appeared to be compacted, forming a bolus consisting of aggregates of starch granules and protein (Fig. 4). For sRB and RCB, the protein/starch matrix instead appeared to have fractured during mastication, resulting in fragments retaining structural features of the original product matrix. In $\mathrm{sRB}$, the fragments appeared more fractured than in RCB. The uRCB bolus also contained fragments, but these mainly consisted of aleurone layers or endosperm cells connected by a weakly stained starch phase, most likely due to hydration. For extR, a similar hydrated starch phase mainly characterised the bolus and, as in WB, the structure appeared to have been compacted into aggregates. RP appeared unaffected by the masticatory process.

After completed gastric digestion, the protein network in WB was completely digested, leaving only free starch granules. For sRB the fragments from the bolus had been mostly disintegrated, while for RCB fragments similar to those observed in the bolus remained. In both uRCB and extR the hydrated starch phase had been disrupted, but the fragments with intact cells in uRCB remained. For RP the effect appeared to be mainly one of dilution. 

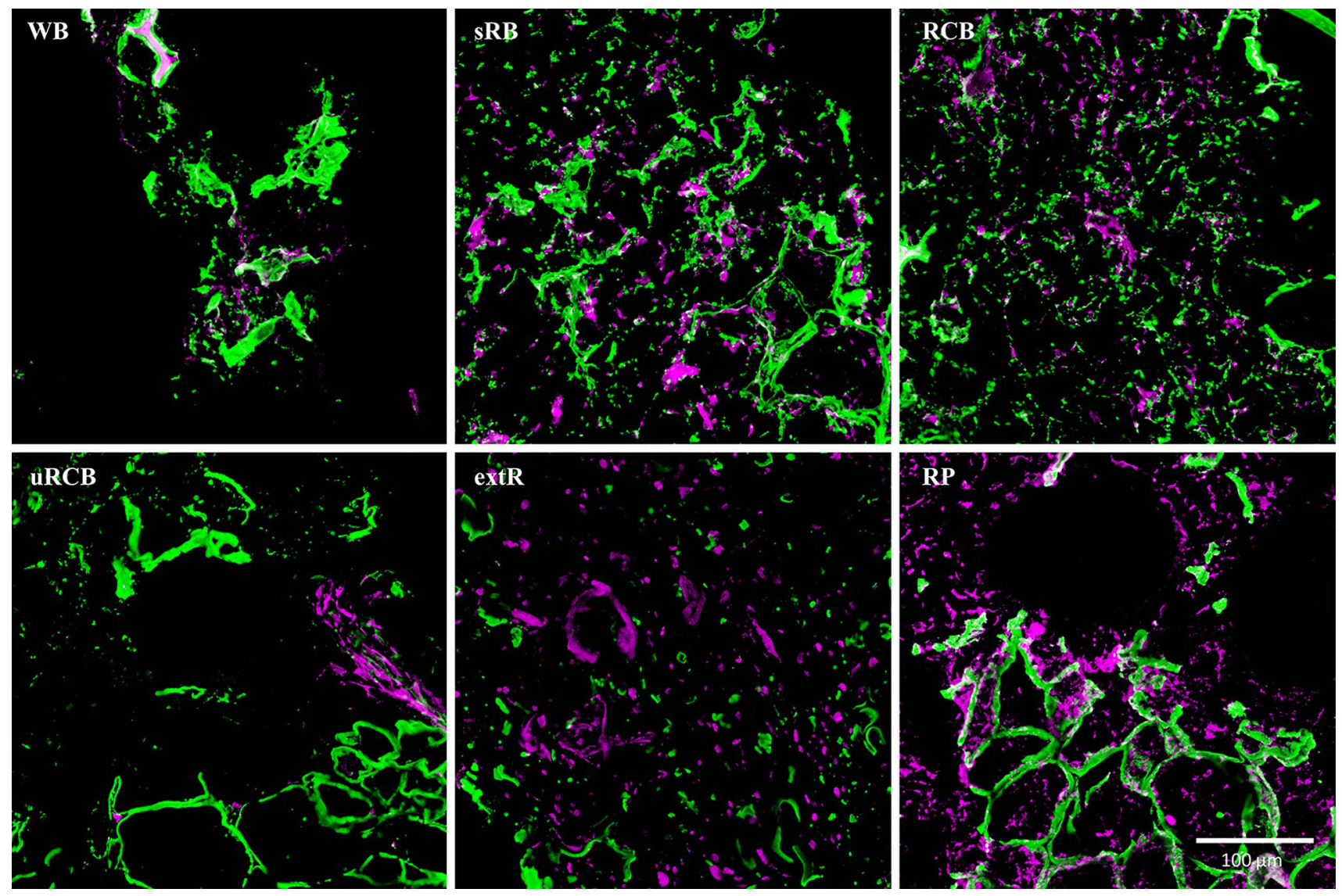

Fig. 2 Confocal laser scanning microscopy micrographs of immunolabelled $\beta$-glucan (green) and arabinoxylan (magenta) in undigested products. $W B$ refined wheat bread. $s R B$ sourdough-fermented whole

\section{Gastric digesta: viscosity}

The viscosity decreased rapidly within the first 5 minutes of simulated gastric digestion in the RVA for all products (Fig. 4). For WB, sRB and RCB, the viscosity then remained stable, while for $\mathrm{URCB}$, extR and RP it increased before stabilising. For all samples peaks could be observed in the viscosity curves. These were most likely caused by bolus fragments too large to pass between the paddle and the cup wall (i.e., $>2 \mathrm{~mm}$ ) and consequently were caught between the paddle and the baffles giving rise to sudden and temporary increase in resistance to mixing. For uRCB and extR, the peaks occurred more frequently and for a longer time, 90 and $50 \mathrm{~min}$, respectively, compared with the other products. The viscosity stabilised most rapidly for sRB, with no peaks observed after $20 \mathrm{~min}$. The final viscosity was higher for $\mathrm{uRCB}$ and RP than for all other products and for extR and RCB compared with WB (Fig. 5). grain rye bread. $R C B$ yeast-fermented whole grain rye crispbread. $u R C B$ unfermented whole grain rye crispbread. ext $R$ extruded whole grain rye. $R P$ whole grain rye porridge

Gastric digesta: particle size distribution

The WB digesta contained the smallest particles, with none above $250 \mu \mathrm{m}$ (Fig. 5). Of the rye products, extR had the smallest particles $(96 \%<250 \mu \mathrm{m})$, followed by sRB, RP and $\operatorname{uRCB}(89,84$ and $83 \%<250 \mu \mathrm{m}$, respectively). RCB contained the highest fraction large particles, with $75 \%$ below $250 \mu \mathrm{m}$.

\section{In vitro digestion of food products in the TIM model}

The glucose concentrations and AUC values differed between the products and there was a statistically significant product $\times$ time interaction (Fig. 6). The AUC value for the whole curve, $\mathrm{AUC}_{0-180 \mathrm{~min}}$, was lower for $\mathrm{WB}$, sRB and $\mathrm{RCB}$ than for RP. For $\mathrm{AUC}_{0-90 \text { min }}$, only sRB was significantly lower than WB $(p<0.05)$. For specific time points, the glucose concentration at $120 \mathrm{~min}$ was higher for RP 


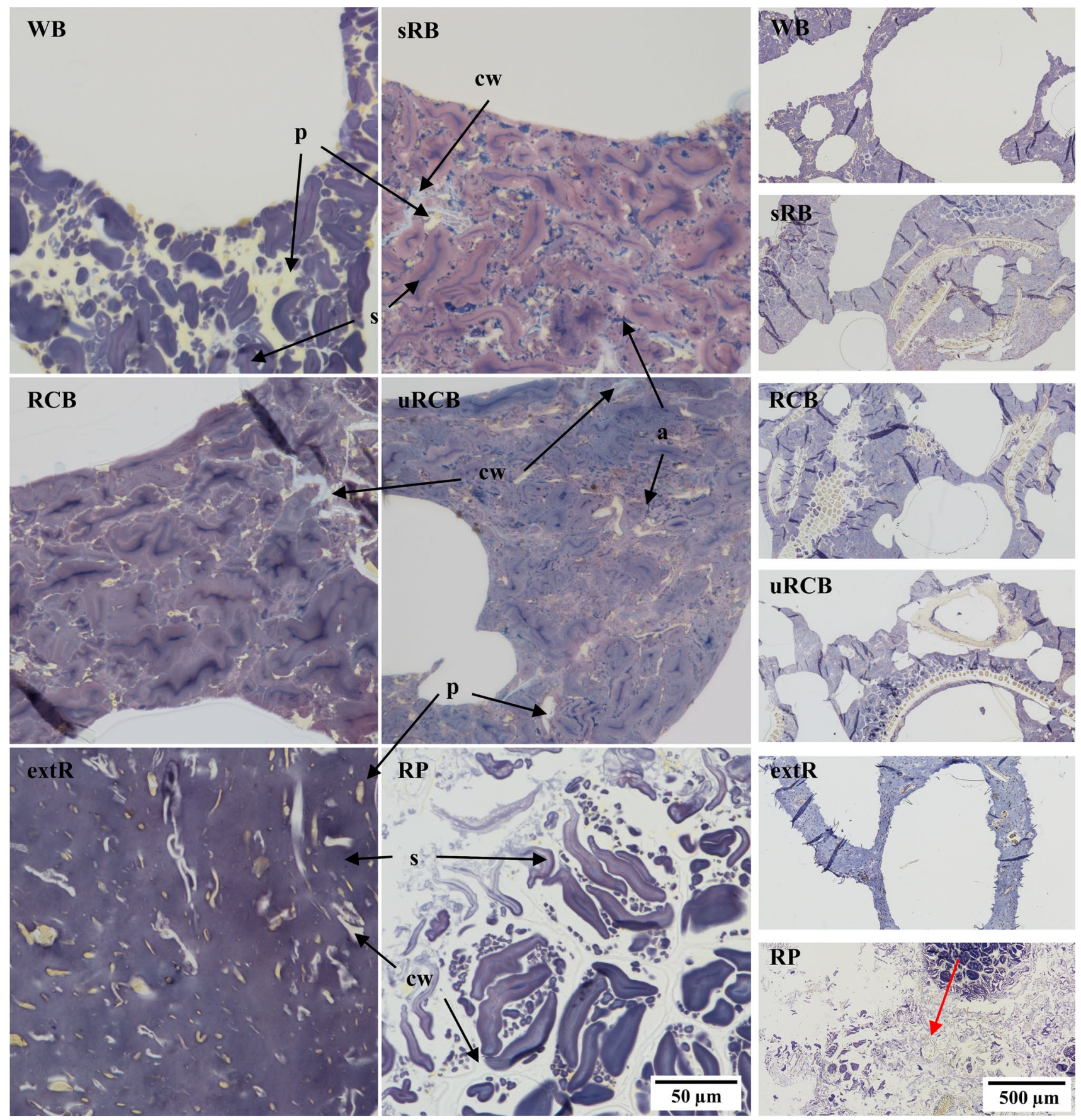

Fig. 3 Light microscopy micrographs stained with iodine showing the microstructure of undigested products at two different magnifications. $W B$ refined wheat bread. $s R B$ sourdough-fermented whole grain rye bread. $R C B$ yeast-fermented whole grain rye crispbread. $u R C B$ unfermented whole grain rye crispbread. extR extruded whole grain

than for all other products and for uRCB and extR compared with WB, sRB and RCB. At $180 \mathrm{~min}$, glucose concentration was higher for $\mathrm{sRB}$, extR and RP compared with WB. For WB and RCB, the peak value occurred at $90 \mathrm{~min}$, while for all other products it occurred at $120 \mathrm{~min}$. rye. $R P$ whole grain rye porridge. Protein $(\mathrm{p})$ is stained yellow, starch (s) purple, amylopectin brown and amylose (a) blue. Cell walls (cw) are unstained, but can be seen in the starch/protein matrix. Arrow (d) indicates transition from intact structure to continuous dilute starch phase in RP

Progressive degradation of the products was observed in samples collected from the duodenal compartment at different time points (Fig. 7). In the WB digesta, the protein/starch aggregates found in the bolus could be observed initially. Over time, these aggregates decreased 

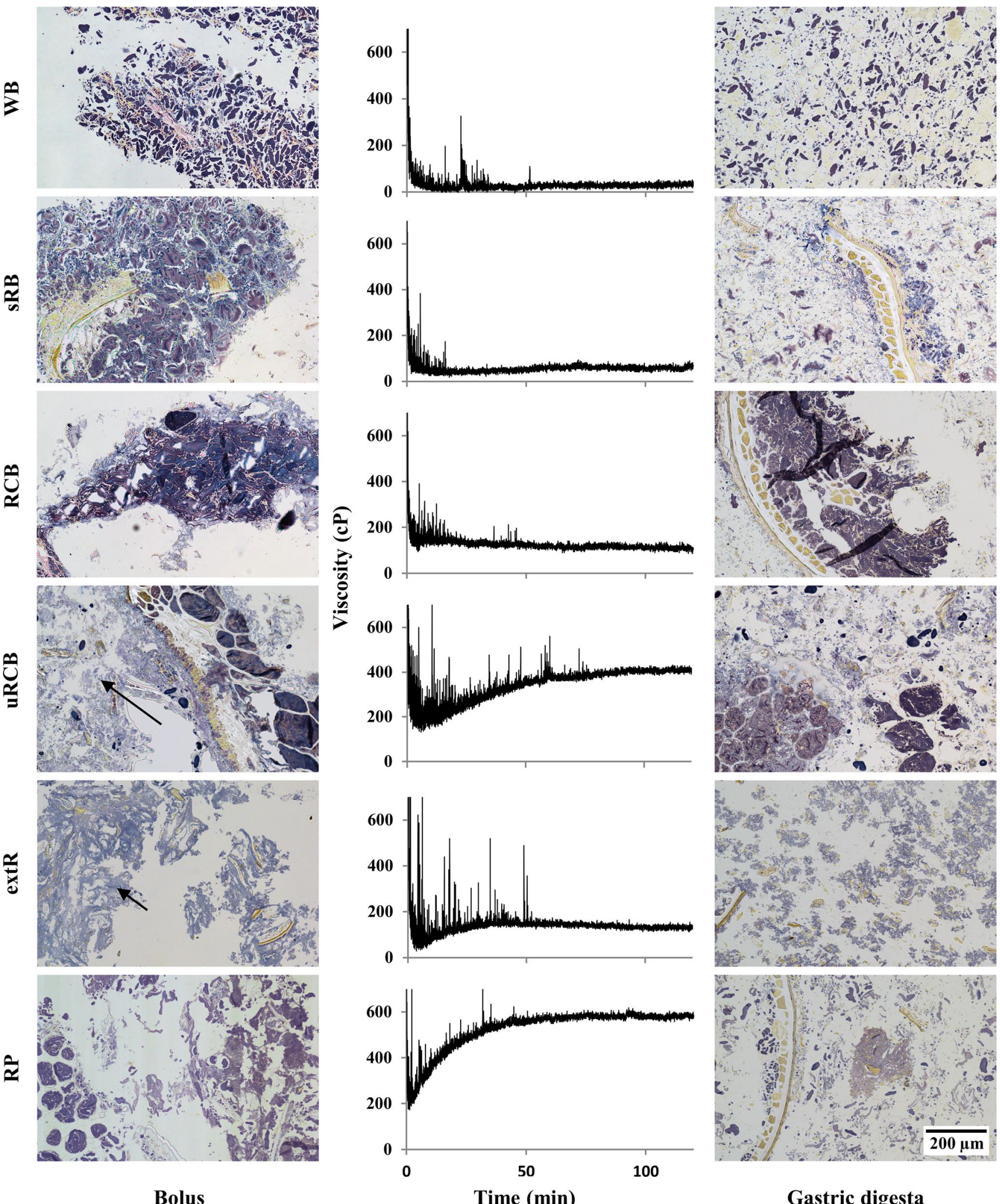

Bolus

Time (min)

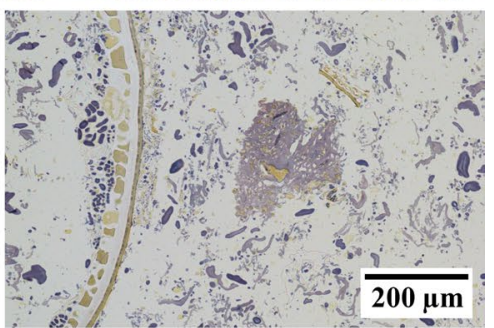

Gastric digesta
Fig. 4 Left Light microscopy micrographs of the masticated samples. Arrows indicate the hydrated starch phase in uRCB and extR. Centre development of viscosity, measured by Rapid Visco Analyser at $120 \mathrm{rpm}$ and $37^{\circ} \mathrm{C}$. Right LM micrographs, $120 \mathrm{~min}$ simulated gastric digestion. Protein is stained yellow, starch purple, amylopectin brown and amylose blue. WB refined wheat bread. $S R B$ sourdoughfermented whole grain rye bread. $R C B$ yeast-fermented whole grain rye crispbread. $u R C B$ unfermented whole grain rye crispbread. extR extruded whole grain rye. $R P$ whole grain rye porridge 

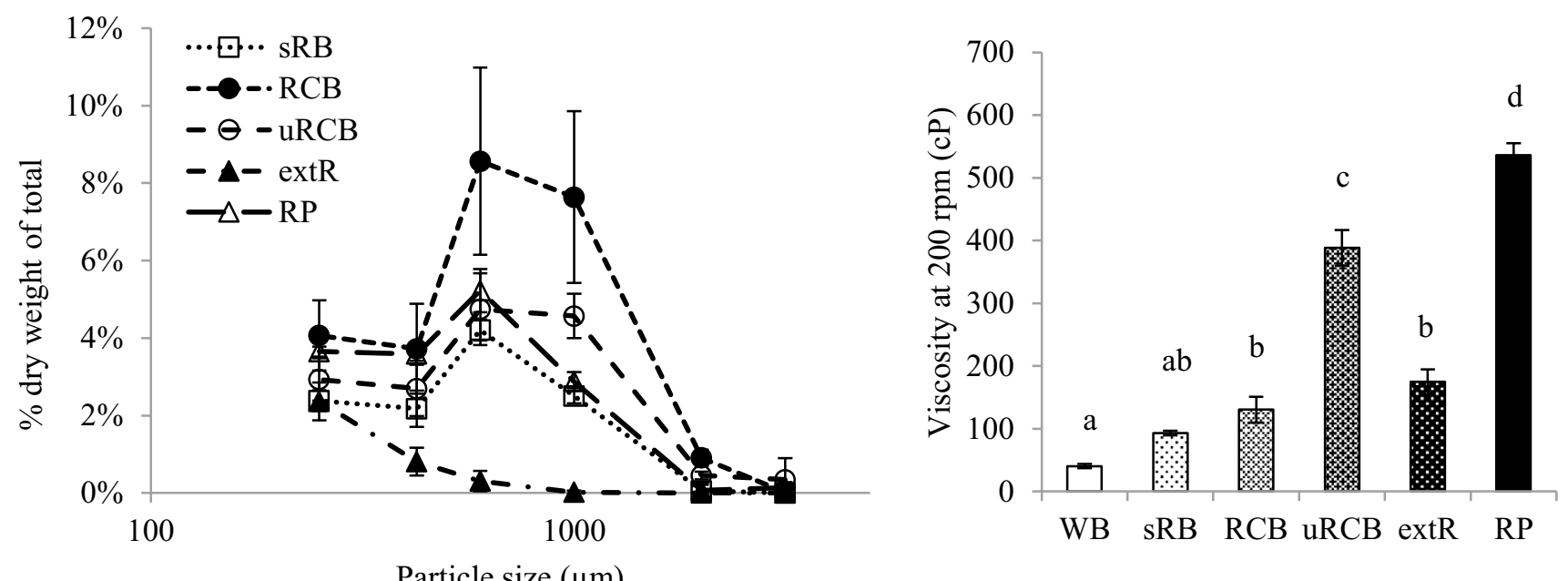

Fig. 5 Left Particle size distribution as a percentage of dry weight of total (mean \pm standard deviation) of particles after static gastric digestion. WB is not included in the graph as all particles were $<250 \mu \mathrm{m}$ in size. Right viscosity of digesta after $120 \mathrm{~min}$ (least square mean \pm standard error) measured at $37^{\circ} \mathrm{C}$ by Rapid Visco
Analyser at $200 \mathrm{rpm}$. Different letters indicate statistically significant differences. $W B$ refined wheat bread. $s R B$ sourdough-fermented whole grain rye bread. $R C B$ yeast-fermented whole grain rye crispbread. $u R C B$ unfermented whole grain rye crispbread. extR extruded whole grain rye. $R P$ whole grain rye porridge
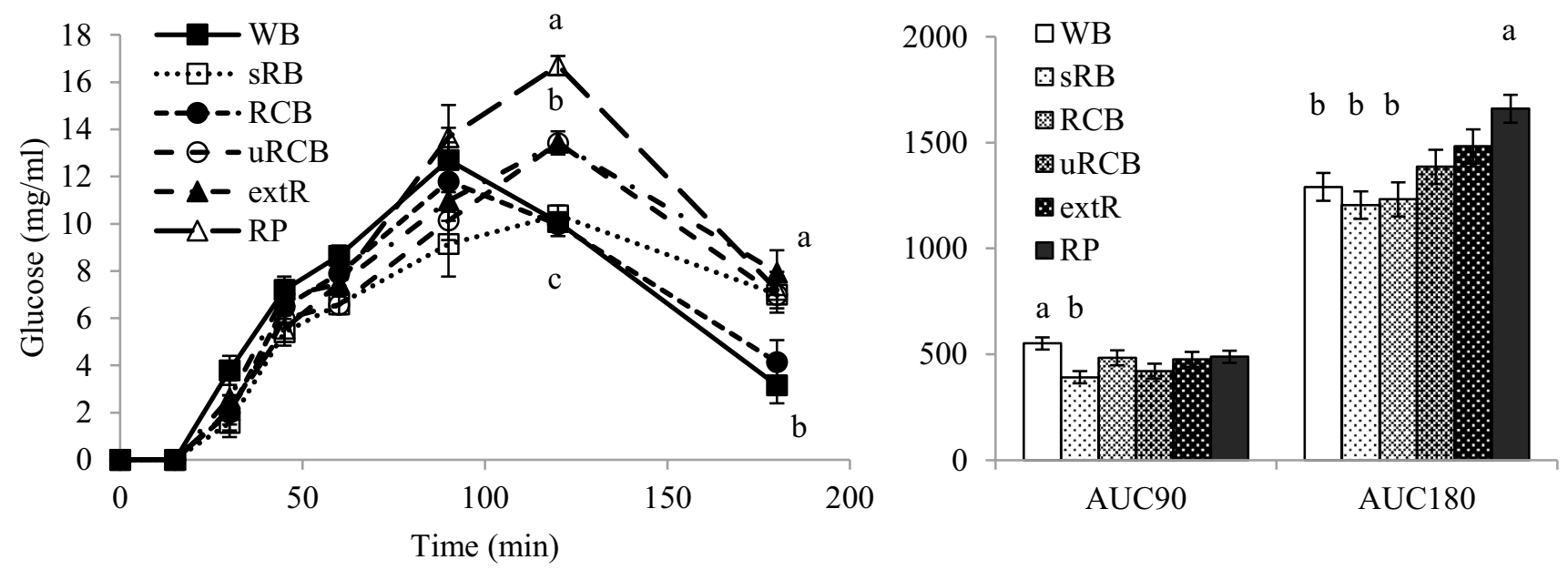

Fig. 6 Glucose concentration profile and area under the curve (AUC). Left Glucose concentration-time profile (mean values). A statistically significant interaction between product $\times$ time was detected $(p<0.05)$. Different letters indicate statistically significant differences between products at certain time points $(p<0.05)$. Right Differences in total $\mathrm{AUC}_{0-180 \min }$ and $\mathrm{AUC}_{0-90 \mathrm{~min}}$ between products.

in size and after 120 min were almost absent. In all rye products except extR, the digestion of starch was observed to proceed from the outside towards the centre of larger fragments. The amount of starch remaining also appeared to decrease with time. Fragments consisting of intact endosperm cells and aleurone layers were observed in $\mathrm{sRB}, \mathrm{RCB}$, uRCB and RP, while in RCB there were also fragments consisting of only starch granules, as
Values are adjusted least square mean \pm standard error. $p<0.05$ was considered significant. $W B$ refined wheat bread. $s R B$ sourdoughfermented whole grain rye bread. $R C B$ yeast-fermented whole grain rye crispbread. $u R C B$ unfermented whole grain rye crispbread. extR extruded whole grain rye. $R P$ whole grain rye porridge

observed after simulated gastric digestion by RVA. In extR, the digesta consisted of notably smaller starch particles than those seen after simulated gastric digestion. In general, the observations from the TIM model were in line with the particle size distribution after 2-h simulated gastric digestion. The main discrepancies were observed for WB, where larger aggregates were initially present, and extR, where the particles appeared smaller. 


\section{Discussion}

In the present work, we found differences in viscosity development, structural disintegration and glucose release during in vitro digestion of rye products depending on processing technique used. These results may contribute to the understanding of mechanisms underlying the beneficial postprandial responses observed for rye products $[4,6]$ and differences in postprandial responses observed for differently processed rye products $[5,7]$.

\section{Influence of product properties on mastication and simulated gastric digestion}

The development of digesta viscosity for the different products will depend on the progressive disintegration of the bolus and hydration, swelling and solubilisation of different components [31]. The rapid initial decline in viscosity and the progressive decrease in frequency and amplitude of peaks was most likely the result of disintegration of the boluses. Although this does not give a direct measure of size or amount of larger fragments the longer duration of occurring peaks could indicate a slower disintegration of the boluses of uRCB and extR (Fig. 4). This could be related to more cohesive boluses resulting from the formation a connective starch phase during mastication, as observed for uRCB and extR (Fig. 4). The thinner lamella and more disrupted and gelatinised starch granules in uRCB and extR compared with sRB and RCB (Fig. 3) may have resulted in structures which were more easily hydrated by saliva during mastication. Plasticisation of the starch phase could give more flexible structures which were compacted during mastication, rather than fractured as appeared to be the case for sRB and RCB (Fig. 4). The continuous protein phase in WB may in a similar way have contributed to a more cohesive bolus compared with sRB and RCB. Refined wheat bread has also been reported to form larger particles after mastication than wholegrain and endosperm rye sourdough bread [32]. The viscosity curve for $\mathrm{SRB}$ seemed to stabilise rapidly compared with the other products, including RCB. This could be related to a weaker structure which was more easily disintegrated. Fractures were also observed in the bolus fragments (Fig. 4). The formation of fractures may have been promoted by less swollen, amylose-surrounded starch granules in sRB. sRB also appeared more disintegrated than RCB after completed gastric digestion (Fig. 4), as reflected in the particle size distribution (Fig. 5). Peaks in the viscosity curve were also observed for the semi-solid $\mathrm{RP}$, indicating presence of larger bolus fragments. As RP was used in a heated state, submersion of the bolus in the colder simulated gastric fluid most likely resulted in gelling of the continuous starch phase and solidification of the bolus which then disintegrated.

In the present work only one individual was used to chew the products and expectorate prior to swallowing (and transfer into the stomach of the dynamic model), since previous investigations have reported that the inter-individual variability of food bolus particle size is very limited, as is the effect of salivary $\alpha$-amylase in relation to the action of pancreatic $\alpha$-amylase [26, 33, 34]. A limitation with mastication is that smaller particles may be lost by "intermediary swallowing" following dispersion in the saliva, and thereby not included in in vitro digestion [35]. Further studies are needed to evaluate and identify key parameters involved in cereal starch digestion and to confirm that the use of one individual to chew is representative.

The observed differences in final viscosity between the products (Fig. 5) may partially relate to fibre composition. Lower molecular weight of $\beta$-glucan (Table 1) and disrupted cell walls (Fig. 2), as observed in SRB and RCB compared with uRCB, extR and RP, is an indication of fibre degradation. $\beta$-glucan is known to be degraded by endogenous enzymes, which become active with the increased moisture content during fermentation [36]. Comparing uRCB with extR and RP, the time needed for mixing and baking of uRCB appeared sufficient for some degradation to occur. Although not analysed, molecular weight of arabinoxylans has been shown to be degraded similarly, but not to the same extent, as $\beta$-glucan [37, 39]. Arabinoxylandegrading enzymes are mainly active at higher temperatures and lower $\mathrm{pH}$, around 4.5 , than $\beta$-glucanases [37, 39], and in $\mathrm{uRCB}$, extR and RP arabinoxylan was probably relatively unaffected. In sRB and RCB; however, some degradation likely occurred. An increase in polydispersity and slight decrease in molecular weight has also been reported for fermented rye crispbreads compared with unfermented [38]. Furthermore, due to lower dough $\mathrm{pH}$, the use of sourdough in SRB might have promoted more extensive degradation of arabinoxylan.

The digesta viscosity is also influenced by the characteristics, e.g., size and shape, of the particles present, although the relationship is not well known [31]. This would explain the relatively low digesta viscosity of extR, despite high extractability and retained molecular weight of $\beta$-glucan and high extractability of arabinoxylan, as it contained the smallest particles of the rye products (Fig. 5).

Viscosity and the rate of disintegration during gastric digestion can influence how rapidly starch becomes available for digestion in the small intestine. Solid foods are considered to be emptied from the gastric compartment first when reaching particle size $<1-2 \mathrm{~mm}$ [40]. Furthermore, in mixed meals, liquids are preferentially emptied first [41]. 


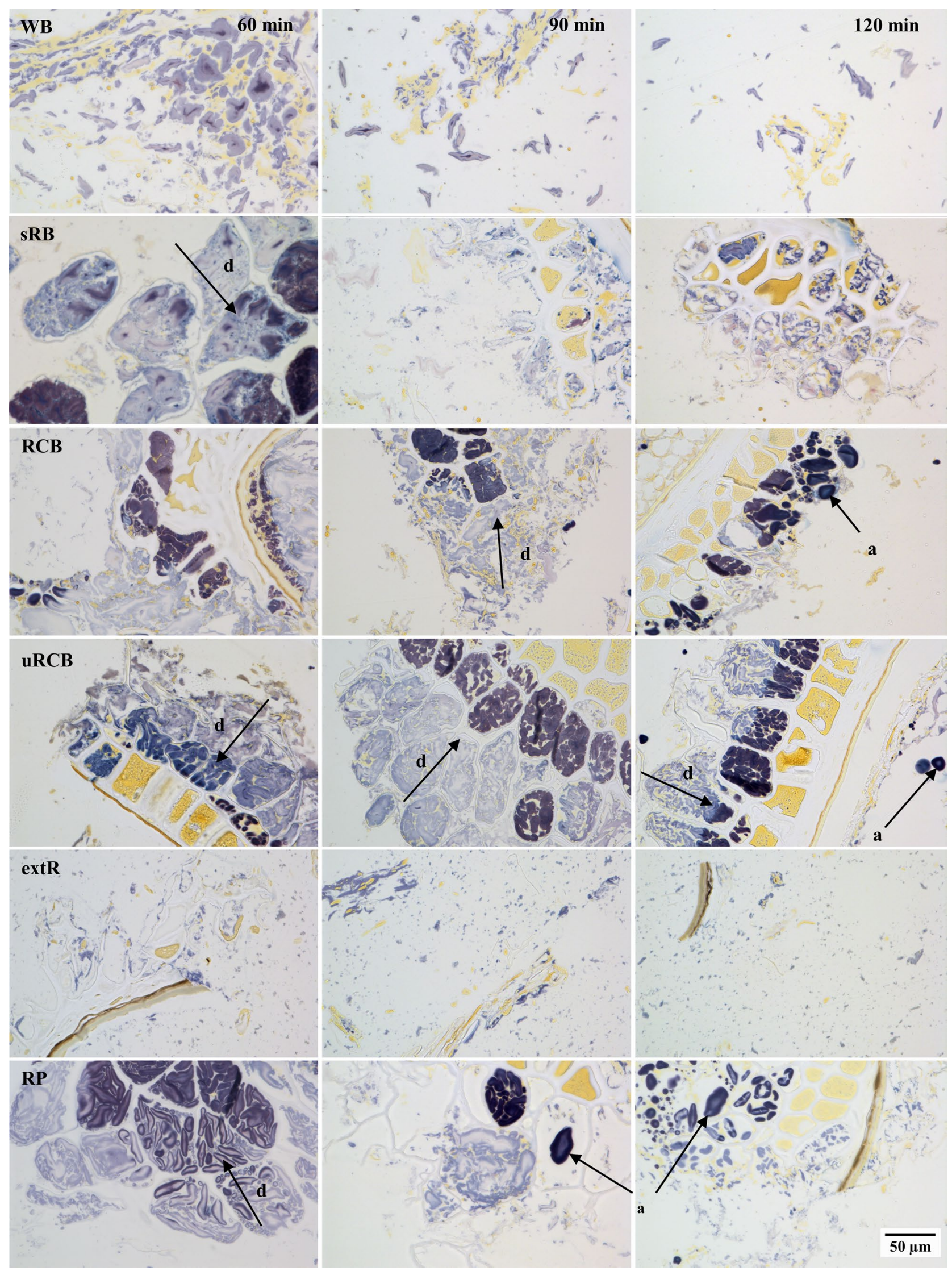


4Fig. 7 Light microscopy micrographs stained with iodine showing the microstructure of samples taken from the duodenal compartment after 60, 90 and 120 min digestion. Arrows marked with d indicate the direction of starch hydrolysis. Ungelatinised starch (a) was seen in RCB, uRCB and RP. $W B$ refined wheat bread. $s R B$ sourdoughfermented whole grain rye bread. $R C B$ yeast-fermented whole grain rye crispbread. $u R C B$ unfermented whole grain rye crispbread. extR extruded whole grain rye. $R P$ whole grain rye porridge

As boluses of cereal foods typically form more cohesive masses than e.g., vegetables [42], the rate of disintegration is likely to be of importance for gastric emptying and consequently the postprandial responses in humans [43]. High viscosity can also contribute to reduced gastric emptying [44]. Furthermore, both content of soluble fibres and viscosity can influence the diffusion rates of enzymes and glucose $[8,9,45]$. During digestion in vivo or in dynamic in vitro models, digesta viscosity will decrease due to continuous dilution by gastric and intestinal juices. However, differences in both viscosity and diffusion rates between products resulting from variations in particle characteristics and fibre composition may still persist.

\section{Influence of product properties on glucose release in the TIM model}

The differences in glucose release between products were not clearly reflected in the progression of starch digestion as observed in the digesta from the TIM model at different time points (Fig. 7). The model has previously been used to visualise differences in starch digestion [17], but in that study a larger difference in total release of maltose was reported and both products tested, oat and barley tempeh, were relatively similar in structure. In our study a range of products with varying structures and properties were used, so different factors may have contributed to the concentration profiles of each product.

It is possible that slower gastric disintegration of $\mathrm{uRCB}$ and extR (Fig. 4) contributed to a later peak in glucose concentration, but similar AUC, compared with WB and RCB by decreasing the rate at which starch became available for digestion. No large bolus fragments $(>2 \mathrm{~mm}$ ) were observed in the intestinal compartments, and gastric sieving appears to occur in the TIM model too, indicating that gastric disintegration will be a factor to consider. Furthermore, lower diffusion rates due to less degraded fibres could decrease the rate of starch hydrolysis and, in the TIM model, reduce the rate of filtration/removal of digested compounds through the dialysis filters. This might also have contributed to the shift in glucose curves observed for uRCB and extR compared with WB and RCB. The highly disrupted starch in extR could have been expected to result in faster starch hydrolysis, but the gastric disintegration and diffusion rates may be more important factors. Despite similar glucose profiles in the TIM model, the responses to uRCB and extR may differ in humans, as the lower digesta viscosity of extR may result in faster gastric emptying rate [39]. The difference in glucose profiles between RCB and $\mathrm{URCB}$ is in line with results of a recent comparison of $\mathrm{RCB}$ and $\mathrm{URCB}$ with a refined wheat crispbread, where RCB produced an insulin response more similar to that of the wheat reference than to $\mathrm{URCB}$ [5].

Differences in the glucose profiles for RCB and WB could have been expected considering the large differences in fibre content and composition, viscosity and particle size distribution. However, at equal viscosities, lower diffusion rates have been demonstrated for solutions of arabinoxylan from wheat compared with arabinoxylan from rye [9]. Moreover, as observed in the TIM model, particles were initially of comparable sizes for $\mathrm{WB}$ and RCB (Fig. 7). While starch hydrolysis rate is often reported to be higher for refined wheat breads than rye breads, measurements are usually made after simulated gastric digestion [4, 6] and the progressive changes in particle sizes occurring in vivo are not accounted for.

For RP, the large product volume, due to its high water content, may have contributed to its high glucose AUC. Compared with the other products, RP occupied a larger fraction of the volume in the gastric compartment and less liquid could be emptied before only the product remained, which should result in faster emptying of product. In humans this may compensate for slower gastric emptying due to high digesta viscosity [44]. Moreover, due to the high water content during preparation and the temperature of the product when initiating in vitro digestion, starch may have been more gelatinised and easily hydrolysed. The high AUC for RP compared with sRB is also in line with findings in a human study by Rosén et al. [7], where endosperm rye porridge and whole grain rye porridge induced higher glucose and insulin responses during the first 30 min than corresponding rye breads.

Neither of the explanations above can account for the low $\mathrm{AUC}_{0-90 \mathrm{~min}}$ and late peak for $\mathrm{sRB}$, with degraded fibres, low digesta viscosity and what appears to be the most rapidly disintegrated bolus of the rye products. However, the results are consistent with the lower insulin or lower insulin and glucose responses compared with refined wheat bread that have been repeatedly shown for soft rye breads, both sourdough-fermented and yeast-fermented [4, 6]. Although lactic acid produced during fermentation has been suggested to inhibit starch hydrolysis, with increased interaction between starch and gluten as the mechanism [46], the absence of a gluten network in sRB indicates other mechanisms. Rather, sRB was the only product where the presence of an amylose layer was observed, which has been suggested to inhibit starch hydrolysis in certain rye products [4]. The high content of resistant starch in sRB 
compared with the other products (Table 1) is likly related to the observed amylose layer (Fig. 3). To our knowledge, the factors contributing to the formation of an amylose layer in certain rye products are not known. However, organic acids produced during sourdough fermentation have been suggested to increase the content of resistant starch and may be a contributing factor [47]. Presence of an amylose layer may also be related to the high water content, compared with RCB, uRCB and extR, which could promote starch retrogradation during storage [48]. Whether the amylose layer is a result of sourdough fermentation or not warrants further investigation.

\section{Conclusions}

From the results of this study, it is apparent that the processing technique affects the hydrolysis of starch and the release of glucose in rye products. Although highly gelatinised starch could be expected to increase the rate of glucose release, in dry products it seems to contribute to formation of a cohesive bolus during mastication which might lead to slower gastric disintegration and glucose release. The importance of different food structures for the bolus formation and the implications this has for the process of gastric disintegration however, need to be further investigated. Fermentation, with yeast or sourdough, leads to degradation of viscous fibres, which could contribute to faster diffusion and gastric emptying rates, increasing the release rate of glucose. However, in the case of rye sourdough bread it may also contribute to the formation of a protective amylose layer around starch granules instead of decreasing release rate. The large meal volume of porridge may contribute to more rapid emptying of product from the gastric compartment. Together with easily available starch, it may lead to faster starch hydrolysis despite high viscosity and intact fibres.

Overall, specific product properties, e.g., starch gelatinisation and fibre degradation, induced by food processing may affect the release rate of glucose in different directions and the net effect may differ between foods. This makes it difficult to draw conclusions on general effects of individual factors when comparing differently processed food products.

Acknowledgements Thanks to Roger Andersson and Gunnel Fransson for their support with fibre analysis and Heliciéné Clément for her assistance with the simulated gastric digestion method.

Authors' contribution DPJ, RL, MA and ML conceived and planned the study and experiments. DPJ conducted the experiments and statistical analysis and drafted the manuscript. JLVG conducted the CLSM experiments. All authors contributed to interpretation of the findings and read and approved the final version of the manuscript.

\section{Compliance with ethical standards}

Conflict of interest On behalf of all authors, the corresponding author states that there is no conflict of interest.

Open Access This article is distributed under the terms of the Creative Commons Attribution 4.0 International License (http://creativecommons.org/licenses/by/4.0/), which permits unrestricted use, distribution, and reproduction in any medium, provided you give appropriate credit to the original author(s) and the source, provide a link to the Creative Commons license, and indicate if changes were made.

\section{References}

1. Ceriello A (2000) The post-prandial state and cardiovascular disease: relevance to diabetes mellitus. Diabetes Metab Res Rev 16:125-132. doi:10.1002/(SICI)15207560(200003/04)16:2<125:AID-DMRR90>3.0.CO;2-4

2. Del Prato S, Leonetti F, Simonson DC, Sheehan P, Matsuda M, DeFronzo RA (1994) Effect of sustained physiologic hyperinsulinaemia and hyperglycaemia on insulin secretion and insulin sensitivity in man. Diabetologia 37:1025-1035

3. Åman P, Andersson AAM, Rakha A, Andersson R (2010) Rye, a healthy cereal full of dietary fiber. Cereal Food World 55:231-234

4. Juntunen KS, Laaksonen DE, Autio K, Niskanen LK, Holst JJ, Savolainen KE, Liukkonen KH, Poutanen KS, Mykkänen HM (2003) Structural differences between rye and wheat breads but not total fiber content may explain the lower postprandial insulin response to rye bread. Am J Clin Nutr 78:957-964

5. Johansson DP, Lee I, Risérus U, Langton M, Landberg R (2015) Effects of unfermented and fermented whole grain rye crispbreads served as part of a standardized breakfast, on appetite and postprandial glucose and insulin responses: a randomized cross-over trial. PLoS One 10:e0122241. doi:10.1371/journal. pone. 0122241

6. Rosén LAH, Östman EM, Björck IM (2011) Effects of cereal breakfasts on postprandial glucose, appetite regulation and voluntary energy intake at a subsequent standardized lunch; focusing on rye products. Nutr J 10:7. doi:10.1186/1475-2891-10-7

7. Rosén LAH, Silva LOB, Andersson UK, Holm C, Östman EM, Björck IM (2009) Endosperm and whole grain rye breads are characterized by low post-prandial insulin response and a beneficial blood glucose profile. Nutr $\mathrm{J}$ 8:42. doi:10.1186/1475-2891-8-42

8. Shelat KJ, Vilaplana F, Nicholson TM, Gidley MJ, Gilbert RG (2011) Diffusion and rheology characteristics of barley mixed linkage $\beta$-glucan and possible implications for digestion. Carbohydr Polym 86:1732-1738. doi:10.1016/j.carbpol.2011.07.004

9. Shelat KJ, Vilaplana F, Nicholson TM, Wong KH, Gidley MJ, Gilbert RG (2010) Diffusion and viscosity in arabinoxylan solutions: implications for nutrition. Carbohydr Polym 82:46-53. doi:10.1016/j.carbpol.2010.04.019

10. Grundy MM, Edwards CH, Mackie AR, Gidley MJ, Butterworth PJ, Ellis PR (2016) Re-evaluation of the mechanisms of dietary fibre and implications for macronutrient bioaccessibility, digestion and postprandial metabolism. Br J Nutr 116:816-833. doi:10.1017/S0007114516002610

11. Björck I, Asp N-G, Birkhed D, Lundquist I (1984) Effects of processing on availability of starch for digestion in vitro and 
in vivo; I extrusion cooking of wheat flours and starch. J Cereal Sci 2:91-103. doi:10.1016/S0733-5210(84)80022-3

12. Holm J, Lundquist J, Björck IME, Eliasson A-C, Asp N-G (1988) Degree in vitro, of starch gelatinization, and metabolic response in rats. Am J Clin Nutr 47:1010-1016

13. Kristensen M, Jensen MG, Riboldi G, Petronio M, Bügel S, Toubro S, Tetens I, Astrup A (2010) Wholegrain vs. refined wheat bread and pasta. Effect on postprandial glycemia, appetite, and subsequent ad libitum energy intake in young healthy adults. Appetite 54:163-169. doi:10.1016/j.appet.2009.10.003

14. Poutanen K, Flander L, Katina K (2009) Sourdough and cereal fermentation in a nutritional perspective. Food Microbiol 26:693-699. doi:10.1016/j.fm.2009.07.011

15. Eelderink C, Moerdijk-poortvliet TCW, Wang H, Schepers M, Preston T, Boer T, Vonk RJ, Schierbeek H, Priebe MG (2012) The glycemic response does not reflect the in vivo starch digestibility of fiber-rich wheat products in healthy men. J Nutr 142:258-263. doi:10.3945/jn.111.147884.into

16. Baggio LL, Drucker DJ (2007) Biology of incretins: GLP-1 and GIP. Gastroenterology 132:2131-2157. doi:10.1053/j. gastro.2007.03.054

17. Alminger MLM, Eklund-Jonsson C, Kidman S, Langton M (2012) Starch microstructure and starch hydrolysis in barley and oat tempe during in vitro digestion. Food Dig 3:53-62. doi:10.1007/s13228-012-0027-8

18. Theander O, Åman P, Westerlund E, Andersson R, Pettersson D (1995) Total dietary fiber determined as neutral sugar residues, uronic acid residues and Klason lignin (the Uppsala method): collaborative study. J AOAC Int 78:1030-1044

19. Loosveld AA, Grobet PJ, Delcour JA (1997) Contents and structural features of water-extractable arabinogalactan in wheat flour fractions. J Agric Food Chem 45:1998-2002

20. McCleary BV, Codd R (1991) Measurment of (1-3), (1-4)- $\beta$-Dglucan in barley and oats: a streamlined enzymic procedure. $\mathrm{J}$ Sci Food Agric 55:303-312

21. McCleary BV, Murphy A, Mugford DC (2000) Measurement of total fructan in foods by enzymatic/spectrophotometric method: collaborative study. J AOAC Int 83:356-364

22. McCleary BV, Monaghan DA (2002) Measurement of resistant starch. J AOAC Int 85:665-675

23. Rimsten L, Stenberg T, Andersson R, Andersson A, Åman P (2003) Determination of $\beta$-glucan molecular weight using SEC with calcofluor detection in cereal extracts. Cereal Chem 80:485-490

24. (1984) Official Journal of the European Communities: determination of crude oils and fat. method B

25. Nordic committee on food analysis nitrogen (1976) determination in food and feed according to Kjeldahl. No 6, Third Ed

26. Ballance S, Sahlstrøm S, Lea P, Nagy NE, Andersen PV, Dessev T, Hull S, Vardakou M, Faulks R (2012) Evaluation of gastric processing and duodenal digestion of starch in six cereal meals on the associated glycaemic response using an adult fasted dynamic gastric model. Eur J Nutr. doi:10.1007/s00394-012-0386-5

27. Loret C, Walter M, Pineau N, Peyron MA, Hartmann C, Martin N (2011) Physical and related sensory properties of a swallowable bolus. Physiol Behav 104:855-864. doi:10.1016/j. physbeh.2011.05.014

28. Minekus M, Alminger M, Alvito P, Ballance S, Bohn T et al (2014) A standardised static in vitro digestion method suitable for food-an international consensus. Food Funct 5:1113-1124. doi:10.1039/c3fo60702j

29. Minekus M, Marteau P, Havenaar R, Huis in't Veld JH (1995) A multicompartmental dynamic computer-controlled model simulating the stomach and small intestine. ATLA Altern Lab Anim 23:197-209

30. Salovaara S, Larsson Alminger M, Eklund-Jonsson C, Andlid T, Sandberg A-S (2003) Prolonged transit time through the stomach and small intestine improves iron dialyzability and uptake in vitro. J Agric Food Chem 51:5131-5136. doi:10.1021/jf0208233

31. Lentle RG, Janssen PWM (2008) Physical characteristics of digesta and their influence on flow and mixing in the mammalian intestine: a review. J Comp Physiol B 178:673-690. doi:10.1007/ s00360-008-0264-x

32. Pentikäinen S, Sozer N, Närväinen J, Ylätalo S, Teppola P, Jurvelin J, Holopainen-Mantila U, Törrönen R, Aura AM, Poutanen $\mathrm{K}$ (2014) Effects of wheat and rye bread structure on mastication process and bolus properties. Food Res Int 66:356-364. doi:10.1016/j.foodres.2014.09.034

33. Woda A, Mishellany-Dutour A, Batier L, Francois O, Meunier J-P, Reynaud B, Alric M, Peyron MA (2010) Development and validation of a mastication simulator. J Biomech 43:1667-1673. doi:10.1016/j.jbiomech.2010.03.002

34. Woolnough JW, Bird AR, Monro JA, Brennan CS (2010) The effect of a brief salivary $\alpha$-amylase exposure during chewing on subsequent in vitro starch digestion curve profiles. Int J Mol Sci. doi:10.3390/ijms11082780

35. Jalabert-Malbos M-L, Mishellany-Dutour A, Woda A, Peyron M-A (2007) Particle size distribution in the food bolus after mastication of natural foods. Food Qual Prefer 18:803-812. doi:10.1016/j.foodqual.2007.01.010

36. Rakha A, Åman P, Andersson R (2011) How does the preparation of rye porridge affect molecular weight distribution of extractable dietary fibers? Int J Mol Sci 12:3381-3393. doi:10.3390/ ijms12053381

37. Isaksson $\mathrm{H}$, Rakha $\mathrm{A}$, Andersson $\mathrm{R}$, Fredriksson $\mathrm{H}$, Olsson J, Åman P (2011) Rye kernel breakfast increases satiety in the afternoon-an effect of food structure. Nutr $\mathbf{J} 10: 31$. doi:10.1186/1475-2891-10-31

38. Rakha A, Åman P, Andersson R (2010) Characterisation of dietary fibre components in rye products. Food Chem 119:859-867. doi:10.1016/j.foodchem.2009.09.090

39. Rasmussen CV, Boskov Hansen H, Hansen $\AA$, Melchior Larsen L (2001) pH-, temperature- and time-dependent activities of andogenous endo- $\beta$-D-xylanase, $\beta$-D-xylosidase and $\alpha$-L-arabinofuranosidase in extracts from ungerminated rye (Secale cereale L.) Grain. J Cereal Sci 34:49-60. doi:10.1006/ jcrs.2000.0376

40. Hellström PM, Grybäck P, Jacobsson H (2006) The physiology of gastric emptying. Best Pract Res Clin Anaesthesiol 20:397407. doi:10.1016/j.bpa.2006.02.002

41. Phillips RJ, Powley TL (2000) Tension and stretch receptors in gastrointestinal smooth muscle: re-evaluating vagal mechanoreceptor electrophysiology. Brain Res Brain Res Rev 34:1-26

42. Tournier C, Grass M, Zope D, Salles C, Bertrand D (2012) Characterization of bread breakdown during mastication by image texture analysis. J Food Eng 113:615-622. doi:10.1016/j. jfoodeng.2012.07.015

43. Phillips LK, Deane AM, Jones KL, Rayner CK, Horowitz M (2014) Gastric emptying and glycaemia in health. Nat Rev Endocrinol 11:112-128. doi:10.1038/nrendo.2014.202

44. Marciani L, Gowland PA, Spiller RC, Manoj P, Moore RJ, Young P, Fillery-Travis AJ (2001) Effect of meal viscosity and nutrients on satiety, intragastric dilution, and emptying assessed by MRI. Am J Physiol Gastrointest Liver Physiol 280:G1227-G1233

45. Wood PJ (2007) Cereal $\beta$-glucans in diet and health. J Cereal Sci 46:230-238. doi:10.1016/j.jcs.2007.06.012

46. Östman EM, Nilsson M, Elmstahl H, Molin G, Björck I (2002) On the effect of lactic acid on blood glucose and insulin responses to cereal products: mechanistic studies in healthy subjects and in vitro. J Cereal Sci 36:339-346. doi:10.1006/ jcrs.2002.0469

47. Liljeberg H, Åkerberg A, Björck I (1996) Resistant starch formation in bread as influenced by choice of 
ingredients or baking conditions. Food Chem 56:389-394. doi:10.1016/0308-8146(95)00199-9

48. Liu Q, Thompson DB (1998) Effects of moisture content and different gelatinization heating temperatures on retrogradation of waxy-type maize starches. Carbohydr Res 314:221-235. doi:10.1016/S0008-6215(98)00310-3 